Planetary Systems in the Universe - Observation, Formation and Evolution

Proceedings IAU Symposium No. 202, (C)2004 IAU

Alan Penny, Pawel Artymowicz, Anne-Marie Lagrange, $\mathcal{G}$ Sara Russell, eds.

\title{
Supportive Evidence for a Brown Dwarf Solar Companion
}

\author{
John J. Matese \\ University of Louisiana at Lafayette, Lafayette, LA 70504-4210, USA
}

\begin{abstract}
We have previously given evidence based on Oort cloud comet orbital elements which suggested that there may be a Jovian-mass brown dwarf in our solar system. An extended cometary database is now available. The analyses have been repeated and we find that the set of statistically significant correlated anomalies is enhanced. We also respond to unsupported objections that have been raised to this conjecture. If real, the wide-binary object would constitute a natural dynamical intermediary between gas giant planetary objects and isolated objects.
\end{abstract}

\section{Introduction}

An enhanced population of Oort cloud cometary major axis orientations which cluster around a "great circle" on the celestial sphere has been detected (Matese, Whitman, \& Whitmire 1999). The orbital properties of these comets are correlated in a way that can most readily be explained as being dynamically produced by a perturber which aids the dominant tidal torque of the galactic disk in making them observable. This can create the observed overpopulation along the arc which amounts to a total increase of $\approx 25 \%$. The possibility that the effect is due to a weak stellar impulse rather than a bound companion is also considered. We review and refute unsupported suggestions that the correlated excess population is due to observational selection effects or bad data. The putative brown dwarf has a mass $\approx 3 \mathrm{M}_{\mathrm{J}}$, a temperature $\approx 200 \mathrm{~K}$ and a mean interaction distance of $\approx 25000 \mathrm{AU}$. The predicted M-band flux is $\approx 2 \times 10^{11} \mathrm{JyHz}$ which will permit direct observation in the infrared by next generation telescopes if the position of the brown dwarf along the arc can be adequately constrained.

\section{Observations and Conclusions}

Matese, Whitman, \& Whitmire (1998) have discussed the question of data selection when the goal is to determine the dynamical mechanisms which make Oort cloud comets $(a>10000 \mathrm{AU})$ observable. They argued that other long-period comet data, or data from comets that are not clearly from the outer cloud because of energy uncertainties, can be excluded since the remaining population is sufficiently large to obtain statistically significant results. They further concluded that there was only one demonstrable observational selection effect for this "class I" data, and that is the self-evident one that comets with large perihelion distances are less likely to be sufficiently well observed over a large portion of their arc so that their orbit determinations would be of high quality. This 


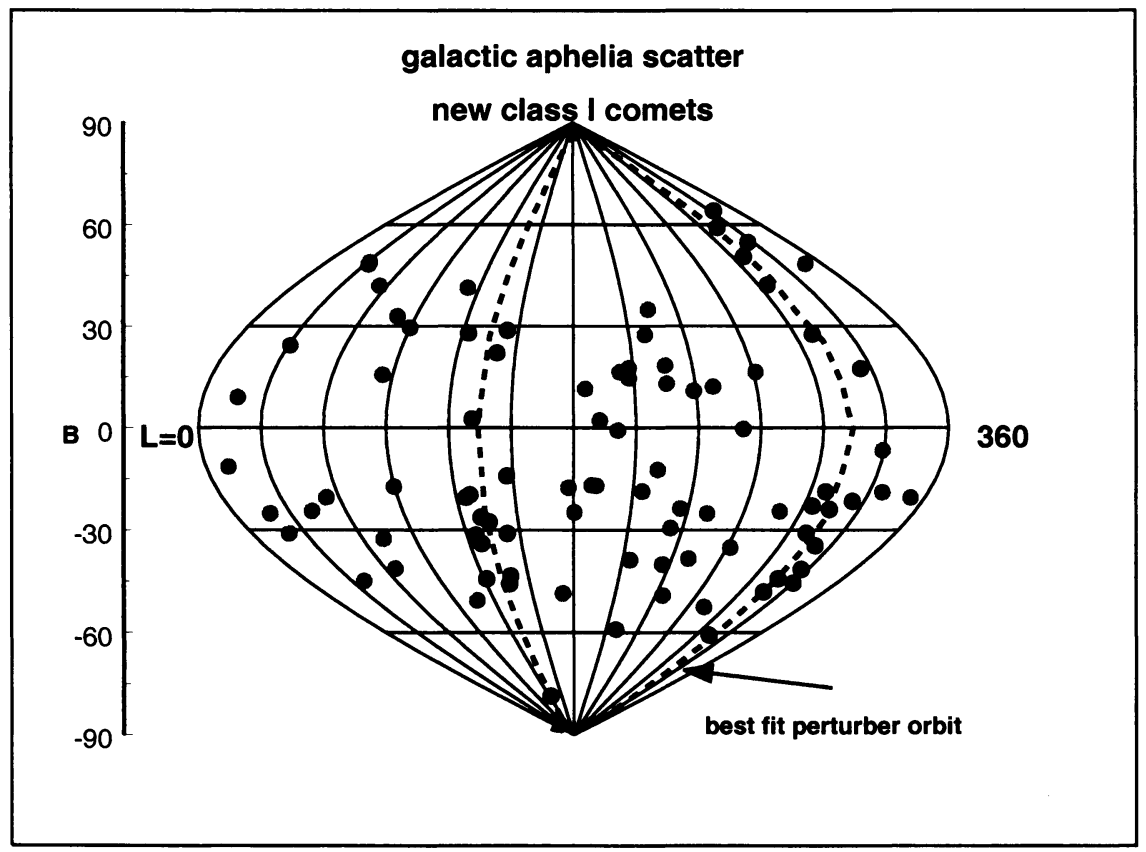

Figure 1. Dark symbols are those with aphelion vectors within 0.2 radians of the best fit companion orbit. These comets are defined as those "inside the great circle band."

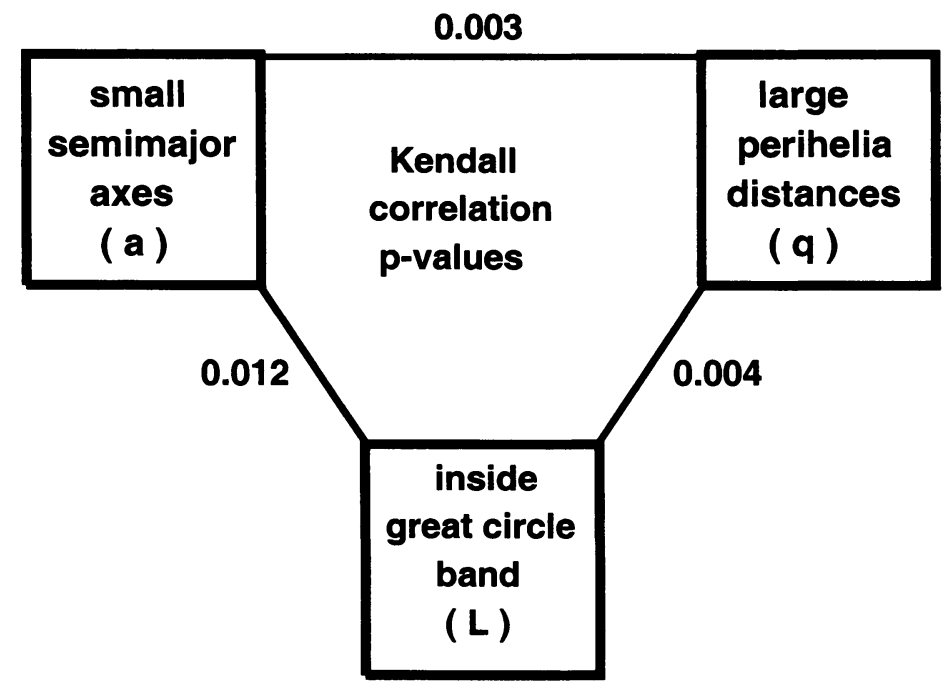

Figure 2. Kendall rank correlation $p$-values are measures of the probability that the dynamical variables would be correlated as much as they are if they were truly unassociated. 
gives a bias against the detection of large- $q$ class I comets. No other selection effect was found to be statistically significant for these data.

We now use an extended database which has increased the number of comets from 82 to 89 (Marsden and Williams 1999) and we repeat the correlation analysis. Specifically, we investigate associations which are a predicted physical consequence of a weak impulsive perturbation aiding the dominant dynamical mechanism that makes Oort cloud comets observable, the tidal torque of the galactic disk ${ }^{1}$. Large $q$ is dynamically associated with small $a$, and in turn they are both associated with comets that are weakly impulsed along the perturber track (Fig. 1). In Fig 2 we show some results of the correlation study. For each pair the $p$-value is substantively reduced from the value found previously.

The dynamical basis for the perturber conjecture has not been challenged, and recently Matese \& Lissauer (2000) have demonstrated that these correlations occur for a stellar impulse. However it was concluded there that the predicted stellar track would be wider and shorter than that observed in Fig. 1. It is now argued that the length and width of the enhanced population is more likely to be caused by a bound Jovian-mass solar companion than by a low mass stellar intruder, and that detailed investigation is in progress.

Unpublished objections raised to this work focus on assertions that the data was "bad", or, that some unspecified observational selection effect caused the correlated enhancement. As yet, no one has suggested how bad data can conspire to create a multiply-correlated enhanced track which accidentally mimics a dynamical effect. In most cases bad data serves to destroy real correlations rather than to spuriously create them. The objections based on selection effects cannot be answered until specificity is given. We note that the only demonstrated selection effect (discussed above) will tend to diminish the correlations found since it selects against large perihelion distances.

The proposed wide-binary gas giant solar companion would constitute a natural dynamical intermediary between simlar objects in planetary orbits (Butler et al, 2001) and in free-floating orbits (Zapatero Osorio et al. 2000).

\section{References}

Butler, R. P. et al., 2001, ASP Conf. Ser. (this volume)

Matese, J. J., \& Lissauer, J. J. 2000 in BAAS 32(3), xx

Matese, J. J., Whitman, P. G., \& Whitmire, D. P. 1998, Cel. Mech. \& Dyn. Ast. 69, 77

Matese, J. J., Whitman, P. G., \& Whitmire, D. P. 1999, Icarus 141, 354

Marsden, B. G., \& Williams, G. V. 1999 in Catalogue of Cometary Orbits 13th edn. Smithsonian Astrophysical Observatory, Cambridge

Zapatero Osorio, M. R. et al. 2000, Science, 290, 103

\footnotetext{
${ }^{1}$ This work is partially supported by a NASA/ASEE Summer Faculty Fellowship, a NSF/AAS International Travel Grant, and a NASA/AAS Small Research Grant.
} 